\title{
L'analyse des visées de la recherche dans la production des mémoires de bachelor de la HEP-BEJUNE : une question d'intérêt professionnel et de culture(s) de recherche(s)?
}

François Joliat HEP-BEJUNE

Francesco Arcidiacono

HEP-BEJUNE
The analysis of research goals in the production of bachelor dissertations at the HEP- BEJUNE: A matter of professional

interest and of research culture(s)?
R ésumé

La formation en recherche suivie par les étudiants des degrés préscolaire et primaire de la Haute école pédagogique des cantons de Berne, Jura et Neuchâtel (HEP-BEJUNE) s'achève par la production d'un mémoire professionnel de niveau bachelor qui vise à développer une posture réflexive. L'objectif de cette étude consiste à identifier les différentes visées de la recherche qui émergent de ces mémoires, afin de mieux déterminer quels sont les intérêts prioritaires des étudiants et si ces intérêts pourraient être orientés en fonction d'éléments contextuels propres à chacun des trois sites de formation dans lequel l'étudiant a suivi sa formation.

Partant de l'hypothèse que ces productions seraient relativement hétérogènes quant à leur visée, nous avons analysé les questions de recherche (QR) d'un échantillon de 48 mémoires, représentatif de l'ensemble des 531 travaux produits par huit cohortes d'étudiants (2004-2011) sur les trois sites de formation de la HEP-BEJUNE (Bienne, Porrentruy et La Chaux-de-Fonds).

Les résultats généraux montrent que les étudiants choisissent en priorité des mémoires à visée disciplinaire ou de gestion des apprentissages. Mais le tri des résultats en fonction des sites de production des mémoires laisse apparaitre qu'à la HEP-BEJUNE une culture intrasite de la recherche semble avoir une influence sur le choix de ces visées, malgré un cursus de formation harmonisé.

\section{Mots-clés}

Professionnalisation des institutions de formation à l'enseignement, analyse des mémoires de bachelor, visées de la recherche, intérêts praxéologiques, culture de recherche par site de formation

Abstract

The final step in research education for pre-service teachers is the production of a dissertation which aims to develop a reflexive posture.

The goal of the present study is to identify the different lines that emerge from these dissertations in order to better determine and orient the education of pre-service teachers on the basis of contextual elements. We analyzed 48 dissertations produced within a curriculum in primary education. The results show the presence of a disciplinary objective or the learning management issue, although an internal culture of research seems to influence the choice of dissertations subjects, despite a harmonized educational curriculum.

\section{Introduction}

En Suisse, depuis plus de dix ans déjà, les exigences de professionnalisation de la formation des enseignants ${ }^{1}$ impliquent que la recherche issue des sciences de l'éducation occupe une place importante dans leur cursus de niveau tertiaire. Comme le mentionne la Conférence suisse des directeurs cantonaux de l'instruction publique (CDIP, 2001), il s'agit «de faire bénéficier les futurs enseignants d'une formation répondant aux exigences élevées que requiert l'exercice de leur profession, notamment en mettant l'accent sur la relation théorie-pratique. Il conviendra en outre [...] d'intégrer la recherche et le développement dans la formation » (p. 2).

En Suisse romande, depuis la création des Hautes écoles pédagogiques (HEP) en 2001, la présence d'une formation en recherche fortement créditée (15 ECTS [European Credits Transfer System]) constitue un élément phare dans le processus de tertiarisation. Pour les étudiants en formation préscolaire et primaire, cette formation s'achève par la production d'un mémoire professionnel de niveau bachelor qui doit refléter un certain nombre de capacités scientifiques - s'approprier des connaissances, des méthodes, des gestes de recherche -, mais aussi des compétences réflexives, pour soutenir un discours construit, fondé sur les multiples facettes du métier d'enseignant. Ces futurs enseignants sont amenés à «développer la capacité d'articuler, de reproduire, d'appliquer ou d'accroître les connaissances, à hauteur des exigences fixées par le niveau d'étude visé »(Joliat, 2011, p.57-58) et à se développer " en tant que sujets et acteurs professionnels " (Vanhulle, 2005 , p. 43). Or, pour une institution de formation, l'évaluation de la production de mémoires réalisés par ses étudiants est aussi un outil privilégié pour s'interroger de manière continue sur la place de la recherche dans ses plans d'étude, la pertinence de ses contenus de formation et de ses produits. 
Dans le présent article, nous jetons un regard critique sur les mémoires de bachelor réalisés depuis la création de la HEP-BEJUNE. Lobjectif de cette étude consiste à identifier les différentes formes de mémoires qui ont été produites par ses étudiants, afin de pouvoir répondre aux questions suivantes :

1. Quelles sont les visées de recherche qui émergent de ces travaux de mémoire?

2. Ces visées se différencient-elles en fonction du site de production des mémoires et quelles en seraient les raisons?

L'analyse des visées de ces mémoires permet d'étudier le mémoire en tant que produit qui achève la formation des futurs enseignants du préscolaire et du primaire, en fonction du site dans lequel il a été rédigé. En première partie, nous développons la question du rôle et des enjeux du mémoire professionnel en HEP. Puis nous présentons le contexte institutionnel dans lequel se situe notre étude. Après un aperçu méthodologique sur le recueil des données, nous faisons état des résultats obtenus pour conclure sur quelques pistes de réflexion.

\section{Contexte}

Plusieurs analyses sur la formation des enseignants ont porté sur les différents dispositifs qui visent à améliorer leur efficacité individuelle et collective, à rationaliser les savoirs mis en œuvre dans l'exercice de leur profession. Ces différents dispositifs basés sur une logique de professionnalisation sont fortement liés aux apports de la recherche en éducation (Clanet, 2010). Quant aux savoirs professionnels, ce sont nécessairement des savoirs composites, "divers types de savoirs puisant à des sources variées de connaissances et de compétences » (Tardif et Borges, 2009, p. 113) qu'il s'agit de subjectiver : « la confrontation à des écarts et l'obligation d'y faire face ou de les comprendre, impliquent des modes nouveaux de raisonner et d'agir, qui engendrent des reconfigurations - ou des autorégulations - à l'intérieur du système mental du sujet »(Vanhulle, 2009a, p. 168-169).

Parmi les productions écrites des étudiants, le mémoire de fin d'études représente un terrain d'observation privilégié pour jauger des effets professionnalisants que peut produire un parcours de réflexion scientifique, transcrit et mis en forme à travers un processus d'écriture longue. Aujourd'hui, après plus de dix ans d'exercice, les HEP mettent en œuvre de nouveaux programmes d'études. Le moment nous paraît donc opportun pour jeter un regard critique sur l'un de ses produits phares : le mémoire de bachelor qui parachève la formation des étudiants inscrits en formation préscolaire et primaire. Une occasion de repenser la place de la recherche et ses produits en formation?

\section{La place du mémoire professionnel dans la formation des enseignants}

Le choix du mémoire professionnel, comme dispositif à analyser dans l'étude de la production de savoirs au sein d'institutions de formation tertiaire, s'inscrit dans une tradition de débats sur ce type spécifique de dispositif de formation à la recherche et par celle-ci. En effet, au cours des années 90 en France, la « controverse » sur les formes, la finalité et les modalités d'accompagnement du mémoire professionnel (Bailleul et Bodergat, 2001) a animé les discussions au sein des Instituts universitaires de formation des maîtres (IUFM) et s'est rapidement propagée à d'autres pays (Cros, 1998). 
Pour pouvoir articuler des savoirs professionnels de nature différente (scientifique et pédagogique) et répondre aux exigences multidimensionnelles de l'exercice de la profession (Lang, 2004), le processus d'universitarisation de la formation à l'enseignement a cristallisé un certain nombre de tensions liées aux enjeux de qualification de la profession (Bourdoncle, 1994), mais aussi à l'intégration de la recherche dans une formation professionnalisante (Tardif et Lessard, 2004), en référence au modèle du praticien réflexif. À ce propos, les travaux de Wentzel (2012), notamment, ont souligné l'importance du lien entre formation, recherche et compétences réflexives dans les dispositifs de formation et dans la conception et la réalisation d'un mémoire professionnel.

La réflexion autour du mémoire professionnel en tant que dispositif de professionnalisation des enseignants comporte plusieurs niveaux d'analyse et une pluralité d'éléments dont il faut tenir compte. Fabre et Lang (2000) distinguent trois tensions constitutives de cet objet de formation :

1. la première résulte du choix de la centration sur le processus ou le produit : la tension entre une formation à la recherche ou par celle-ci, en référence au paradigme de l'apprenti chercheur ou à celui du praticien réflexif;

2. la deuxième concerne le statut de l'écriture : la tension entre sa fonction instrumentale d'exposition d'une pensée déjà constituée (le rapport de recherche) et la trace d'une « aventure de conscientisation » (le mémoire professionnel) (p. 52);

3. la troisième provient du caractère identitaire du mémoire : la tension entre une trajectoire personnelle et la socialisation professionnelle.

En formation professionnelle, l'expérience de réalisation d'un mémoire devient significative, lorsqu'elle aboutit « à la connaissance fine d'un objet en lien avec la pratique, à la construction de ressources pour observer et comprendre le réel [...] et à la structuration d'une identité de praticien réflexif » (Wentzel, 2010, p. 167). La formation à la recherche et par celle-ci constitue une modalité privilégiée de production de savoirs sur les professions et pour celles-ci (Hofstetter et Schneuwly, 2009). Elle offre un accompagnement dans la structuration d'une pensée réflexive qui mobilise des ressources, des outils guidant la réflexion et, plus globalement, un savoir-analyser (Altet, 2000). Or, ces savoirs sont souvent des savoirs composites :

La construction des savoirs professionnels ne dépend pas que de la formation académique qui doit composer avec des références multiples. Avec les savoirs savants et scientifiques coexistent des prescriptions de l'employeur, des pratiques transmises par des formateurs de terrain et des expériences situées dans des contextes de stages. (Vanhulle, 2009b, p. 246)

Cependant, au sein des HEP s'est rapidement dessinée une logique de recherche pédagogique pragmatique, liée à des impératifs praxéologiques (Hofstetter, 2005), qui a déterminé le contexte dans lequel le mémoire professionnel devait s'inscrire : un instrument et un outil de formation supplémentaires, permettant aux étudiants de mieux guider leur action pédagogique dans un contexte directement utilisable.

Un autre aspect à prendre en compte dans la réalisation d'un mémoire est lié à la réflexion autour de l'identité professionnelle que la réalisation d'un tel travail de recherche peut renforcer (Cros, 1998; Heer et Akkari, 2005; Perrin, Bétrix Köhler, Martin et Baumberger, 2008). Le mémoire est l'une des 
mises en œuvre du modèle réflexif où l'enseignant s'imprègne de sa profession en prenant du recul, en problématisant ses gestes professionnels, en observant ses collègues et ses élèves, en démontrant sa capacité à trouver des éclairages possibles dans les théories et dans l'analyse de pratiques observées. Le mémoire devient donc un "nouveau genre " (Crinon, Marin, Rayou et Ricard-Fersing, 2005) qui se caractérise par un but spécifique et entraîne à analyser les situations professionnelles pour faire émerger la complexité de la pratique enseignante. L'intention d'une « mise en mouvement » (Wittorski, 2008, p. 70) des futurs enseignants dans des systèmes d'action traduit cette offre de professionnalisation, à partir du moment où le mémoire construit et rend visible ce parcours de réflexion et d'action.

\section{Contexte institutionnel de l'étude}

Sur le plan organisationnel, la HEP-BEJUNE est née de la volonté des Départements de l'instruction publique des cantons de Berne (pour sa partie francophone), du Jura et de Neuchâtel de fusionner leurs trois établissements cantonaux de formation des maîtres (Écoles normales des cantons de Berne et Neuchâtel, Institut pédagogique jurassien du canton du Jura) en une nouvelle institution tricantonale de niveau tertiaire. Or, "la HEP-BEJUNE n'a pas été créée en opérant une rupture totale avec les anciennes institutions. Ainsi, l'ensemble du personnel a été repris et rares ont été les engagements de formateurs extérieurs »(HEP-BEJUNE, 2006, p. 10). Le rapport quinquennal 2001-2006 du Rectorat décline leurs profils professionnels selon deux spécialisations ${ }^{2}$ :

1. orientation didactique. Ce profil est très largement majoritaire chez les formateurs de la HEPBEJUNE. Il concerne des enseignants généralistes et/ou spécialistes ayant été formés dans les anciennes Écoles normales, à l'Université ou dans des Hautes écoles de métiers ou d'arts, dont la plupart ont une longue expérience de formateurs de terrain. Ils enseignent la didactique d'une ou plusieurs disciplines, parfois la didactique générale ou d'autres matières liés à l'enseignement;

2. orientation sciences de l'éducation. Ce profil est minoritaire chez les formateurs de la HEPBEJUNE. Il concerne des enseignants généralistes et/ou spécialistes du niveau préscolaire ou primaire, mais avec une formation universitaire supplémentaire en sciences de l'éducation.

C'est donc dans un contexte forgé par trois histoires institutionnelles longues de plusieurs décennies et d'histoires professionnelles de formateurs affiliés depuis longtemps à leur institution d'origine, qu'une identité de la recherche a dû émerger, se construire, s'affirmer et s'opérationnaliser dans des curricula communs, mis en œuvre par des formateurs au profil professionnel très contrasté.

Dans les orientations générales de la HEP-BEJUNE concernant la place du mémoire dans la formation des enseignants, «le mémoire permet une approche raisonnée de la pratique professionnelle par l'utilisation d'une démarche de recherche fondée sur des méthodes et des outils scientifiques » (HEPBEJUNE, 2010). Il peut générer de nombreuses variantes d'écrits, notamment, par l'encouragement «d'une forme de créativité méthodologique »(Perrin, Bailleul et Bodergat, 2009, p. 19). 


\section{Échantillon}

Entre l'année 2004 et l'année 2011, huit filières d'étudiants des trois sites de formation de la HEPBEJUNE, le site de Porrentruy (SPO), celui de Bienne (SBI) et celui de La Chaux-de-Fonds (SCF) ont produit 531 mémoires, réalisés en solo ou en duo.

Par tirage au sort, nous avons constitué un échantillon de 48 mémoires (9\% de l'effectif total), représentatif du nombre de mémoires produits par chaque site pour l'ensemble des huit filières étudiées (voir Tableau 1) ${ }^{3}$. Sur le site de Porrentruy (SPO), nous avons sélectionné 10 travaux (20,8 \% de l'échantillon) sur les 109 mémoires produits (20,5\% de la production totale). Sur le site de Bienne (SBI), nous avons sélectionné 14 travaux (29,2\% de l'échantillon) sur les 150 mémoires produits (28,3\% de la production totale) et sur le site de La Chaux-de-Fonds (SCF), nous avons sélectionné 24 travaux (50\% de l'échantillon) sur les 272 mémoires produits (51,2\% de la production totale).

\section{Tableau 1}

Production des mémoires de bachelor de la HEP-BEJUNE par année de promotion et par site $(N=531)$ et clé de répartition de l'échantillonnage $(\mathrm{n}=48)$.

\begin{tabular}{|l|l|l|l|l|l|l|l|l|}
\hline $\begin{array}{l}\text { Année de } \\
\text { promotion }\end{array}$ & $\mathrm{SPO}$ & $\mathrm{N}$ & $\mathrm{n}$ & $\mathrm{N}$ & $\mathrm{N}$ & $\mathrm{SCF}$ & \multicolumn{2}{|c|}{ Total de mémoires } \\
\hline $\mathbf{2 0 0 4}$ & 19 & 1 & 11 & 1 & 28 & 3 & 58 & 5 \\
\hline $\mathbf{2 0 0 5}$ & 19 & 1 & 41 & 4 & 0 & - & 60 & 5 \\
\hline $\mathbf{2 0 0 6}$ & 13 & 1 & 19 & 1 & 45 & 2 & 77 & 4 \\
\hline $\mathbf{2 0 0 7}$ & 10 & 1 & 20 & 1 & 48 & 2 & 78 & 4 \\
\hline $\mathbf{2 0 0 8}$ & 12 & 1 & 16 & 2 & 38 & 7 & 66 & 10 \\
\hline $\mathbf{2 0 0 9}$ & 15 & 1 & 15 & 2 & 39 & 5 & 69 & 8 \\
\hline $\mathbf{2 0 1 0}$ & 7 & 1 & 16 & 1 & 32 & 2 & 55 & 4 \\
\hline $\mathbf{2 0 1 1}$ & 14 & 3 & 12 & 2 & 42 & 3 & 68 & 8 \\
\hline $\begin{array}{l}\text { Total } \\
\text { de mémoires } \\
\text { par site }\end{array}$ & 109 & 10 & 150 & 14 & 272 & 24 & 531 & 48 \\
\hline $\begin{array}{l}\text { \% de mémoi- } \\
\text { res } \\
\text { par site }\end{array}$ & 20,5 & 20,8 & 28,3 & 29,2 & 51,2 & 50 & 100 & 100 \\
\hline \begin{tabular}{l} 
Écart en \% \\
\hline
\end{tabular} & +.3 & & +.9 & & $-1,2$ & & & \\
\hline
\end{tabular}

Les proportions des écarts entre le nombre de mémoires choisis pour l'échantillon et le nombre de mémoires de la production totale par site sont peu importantes $(\mathrm{SPO}=.3 \%$; $\mathrm{SBI}=.9 \%$ et $\mathrm{SCF}$ : $1.2 \%$ ) (voir Tableau 1), ce qui nous fait dire que le principe de proportionnalité entre l'échantillonnage par site et la production totale est bien respecté. 


\section{Méthode}

Une méthode d'analyse qualitative/quantitative mixte (Arcidiacono et De Gregorio, 2008) a été employée pour identifier les traces des visées de la recherche dans les mémoires de l'échantillon, à partir de la Question de recherche (QR) (voir Tableau 2).

\section{Tableau 2}

Les visées de la recherche selon sept catégories conceptualisantes: exemples de thématiques et de questions de recherche.

\begin{tabular}{|c|c|c|}
\hline Visé(e)s de la recherche & Descriptif & Thématique et question de recherche (QR) \\
\hline Disciplinaire & $\begin{array}{l}\text { Vise à étudier, comprendre et amélio- } \\
\text { rer des méthodes d'enseignement, des } \\
\text { processus didactiques et des perfor- } \\
\text { mances dans une discipline scolaire } \\
\text { donnée }\end{array}$ & $\begin{array}{l}\text { Thématique : L'orthographe } \\
\text { QR: } \\
\text { Est-ce que l'on observe une progression de la maitrise } \\
\text { orthographique chez les élèves lorsqu'ils peuvent } \\
\text { s'aider d'un outil de référence créé par eux, sur la base } \\
\text { de leurs erreurs? [Fjo6] }\end{array}$ \\
\hline $\begin{array}{l}\text { Gestion des } \\
\text { apprentissages }\end{array}$ & $\begin{array}{l}\text { Vise à étudier, comprendre et amé- } \\
\text { liorer des processus de gestion des } \\
\text { apprentissages (autonomie, évalua- } \\
\text { tion, autodétermination, etc.) }\end{array}$ & $\begin{array}{l}\text { Thématique : L'échec scolaire } \\
\text { QR: } \\
\text { Quels sont les représentations et les aménagements } \\
\text { pédagogiques des enseignants face à des élèves en } \\
\text { situation d'échec scolaire? [Ber8] }\end{array}$ \\
\hline Gestion de la classe & $\begin{array}{l}\text { Vise à étudier, comprendre et } \\
\text { améliorer des processus de gestion } \\
\text { de la classe (interaction, égalité des } \\
\text { chances, travaux de groupe, autorité, } \\
\text { sanction, etc.) }\end{array}$ & $\begin{array}{l}\text { Thématique : La sanction éducative } \\
\text { QR: } \\
\text { Quels sont les liens entre les conceptions des élèves } 5 \\
\text { P et celles des enseignants au sujet de la sanction } \\
\text { éducative? [Ber19] }\end{array}$ \\
\hline $\begin{array}{l}\text { Traits spécifiques des } \\
\text { élèves }\end{array}$ & $\begin{array}{l}\text { Vise à étudier, comprendre et amélio- } \\
\text { rer des comportements, des conditions } \\
\text { d'existence, des traits cognitifs d'élèves } \\
\text { particuliers }\end{array}$ & $\begin{array}{l}\text { Thématique : L'enfant surdoué } \\
\text { QR: } \\
\text { Quels moyens utiliser pour encadrer au mieux un } \\
\text { enfant surdoué? [Fjo19] }\end{array}$ \\
\hline $\begin{array}{l}\text { Besoins spécifiques des } \\
\text { élèves du point de vue } \\
\text { de l'éducation spécia- } \\
\text { lisée }\end{array}$ & $\begin{array}{l}\text { Vise à étudier, comprendre et } \\
\text { améliorer des comportements, } \\
\text { des conditions d'existence, des } \\
\text { traits cognitifs d'élèves à inclure en } \\
\text { classe }\end{array}$ & $\begin{array}{l}\text { Thématique : L'enfant trisomique } \\
\text { QR: } \\
\text { Quels moyens mettre en œuvre pour inclure un } \\
\text { enfant porteur de trisomie } 21 \text { dans une classe } \\
\text { ordinaire du canton de Neuchâtel? [Fra14] }\end{array}$ \\
\hline
\end{tabular}




\begin{tabular}{|c|c|c|}
\hline $\begin{array}{l}\text { Besoins spécifiques } \\
\text { du point de vue de } \\
\text { l'intégration cultu- } \\
\text { relle (allophones, autres } \\
\text { cultures) }\end{array}$ & $\begin{array}{l}\text { Vise à étudier, comprendre et } \\
\text { améliorer des comportements, des } \\
\text { conditions d'existence, des traits } \\
\text { cognitifs d'élèves à intégrer en } \\
\text { classe }\end{array}$ & $\begin{array}{l}\text { Thématique : Les conflits ethniques en milieu } \\
\text { scolaire } \\
\text { QR : } \\
\text { Dans quelle mesure l'éducation aux citoyennetés, } \\
\text { définie par le PER, permet-elle aux enseignants } \\
\text { de régler des conflits à caractère ethnique au sein } \\
\text { de leur classe? [Ber2] }\end{array}$ \\
\hline $\begin{array}{l}\text { Structures, pro- } \\
\text { grammes, habitudes } \\
\text { éducatives }\end{array}$ & $\begin{array}{l}\text { Vise à étudier des structures, des } \\
\text { programmes des habitudes qui ré- } \\
\text { gissent les organisations scolaires } \\
\text { et le monde de l'éducation }\end{array}$ & $\begin{array}{l}\text { Thématique : Relations parents-enseignants } \\
\text { QR : } \\
\text { L'entretien individuel d'évaluation est-il un moyen } \\
\text { essentiel pour l'enseignant d'informer les parents } \\
\text { d'élèves? [Ber9] }\end{array}$ \\
\hline
\end{tabular}

En méthodologie de la recherche (Quivy et van Campenhoudt, 1995), la QR est la clé de voûte sur laquelle repose l'ensemble du processus de recherche. Étayée par les éléments théoriques fournis dans la partie Problématique, parfois synthétisés dans un Cadre théorique, et souvent précédée d'un Modèle d'analyse (Giroux et Tremblay, 2009), la QR doit renseigner sur « le point précis sur lequel la recherche interroge la réalité » (Lamoureux, 2006, p. 107). Sa formulation explicite, méthodologiquement parlant, la manière dont l'auteur entend décliner les phénomènes à produire, en indicateurs de recherche opérationnalisables: «les signes empiriques de dimensions ou de composantes » (Dépelteau, 2000, p. 180). Elle est suivie d'hypothèses que les résultats à venir permettront de confirmer ou d'infirmer ou d'objectifs qui décriront la manière dont l'auteur entend répondre à sa question de recherche.

Dans notre procédé, une grille d'analyse des traces des visées de la recherche a été construite sur la base de sept catégories conceptualisantes définies a priori (Paillé et Mucchielli, 2008) (voir Tableau 2): Disciplinaire, Gestion des apprentissages, Gestion de la classe, Traits spécifiques des élèves, Besoins spécifiques (pédagogie spécialisée), Besoins spécifiques (intégration) et Structures, programmes et habitudes éducatives. Une évaluation qualitative interjuges de ces traces a permis d'identifier la nature de la visée exprimée par chaque mémoire, en fonction de son assimilation à une des sept catégories. Cette évaluation a ensuite été reportée dans la grille en termes de présence/absence par un codage quantitatif binaire (1/0). Onze mémoires $(22,9 \%$ de l'échantillon) contenaient plusieurs visées. Ils ont été codés dans deux $(.5 ; .5)$, voire trois catégories $(.3 ; .3 ; .3)$. Mais le score total du codage de ces visées multiples a toujours été de (1). Les résultats présentés ci-dessous expriment par conséquent l'importance de chaque visée sur l'ensemble de l'échantillon, tous mémoires confondus $(n=48)$.

\section{Résultats}

Nous avons ensuite calculé la fréquence d'apparition des visées de la recherche pour l'ensemble de l'échantillon des mémoires de bachelor produits par les futurs enseignants de la HEP-BEJUNE, tous sites confondus, puis nous les avons classées par ordre d'importance (voir Figure 1). 


\section{Les visées de la recherche sur l'ensemble des trois sites de production des mémoires}

Les visées Disciplinaire $(23,96 \%)$ et Gestion des apprentissages $(23,6 \%)$ sont les plus nombreuses. Suivent ensuite Traits spécifiques des élèves (16,67\%), Structures, programmes et habitudes éducatives $(15,27 \%)$ et Gestion de la classe (12,15\%). Enfin, Besoins spécifiques (intégration) (6,25\%) et Besoins spécifiques (pédagogie spécialisée) (2,08 \%) figurent en fin de classement.

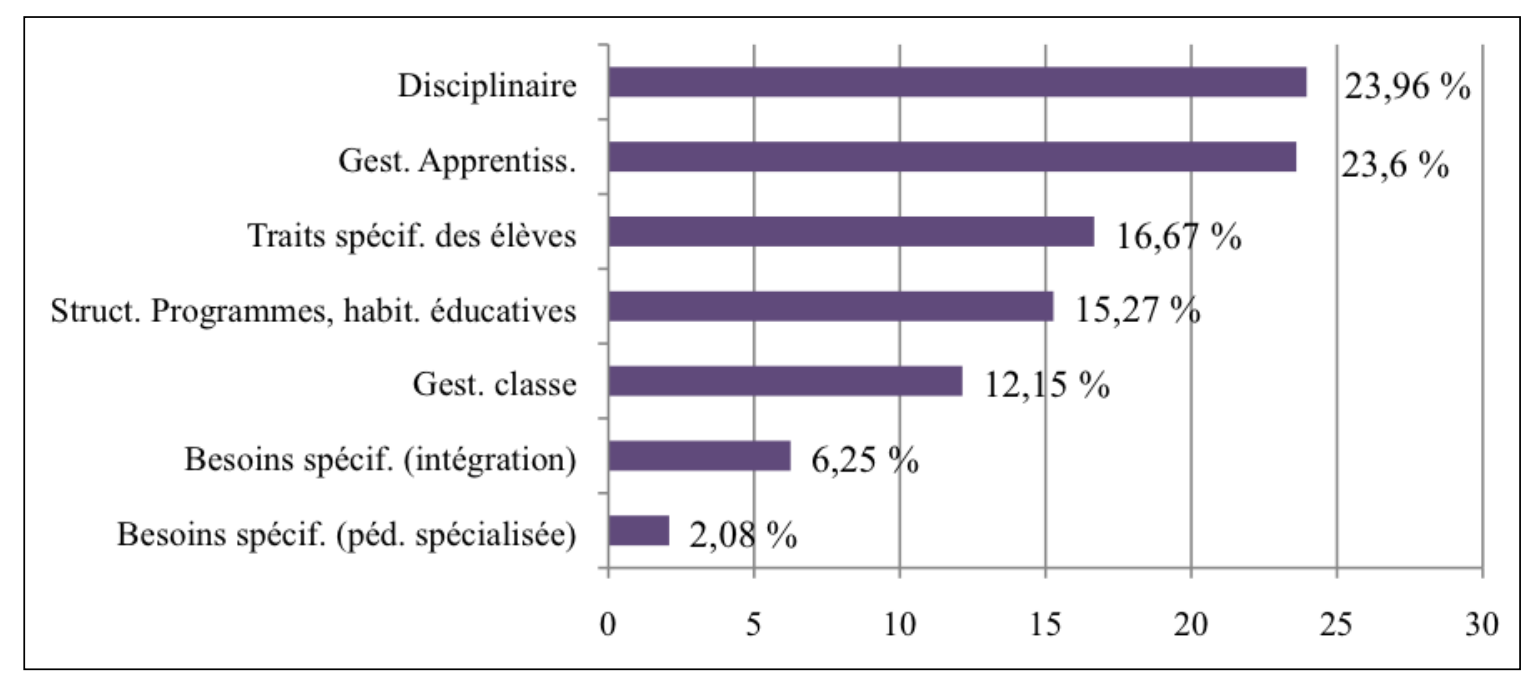

\section{Figure 1}

Classement des fréquences d'apparition des visées de la recherche dans l'échantillon $(n=48)$ des mémoires de bachelor de la HEP-BEJUNE.

\section{Discussion}

Nous voyons deux interprétations possibles au sujet de la fréquence élevée de mémoires à visée Disciplinaire et à visée de Gestion des apprentissages. La première est d'ordre «économique et managérial ». Nous formulons l'hypothèse qu'en fonction d'un calcul de rapport " effort consenti/rentabilité », les étudiants choisissent de s'engager en plus grand nombre dans un mémoire à visée disciplinaire ou qui traite de la gestion des apprentissages, parce qu' ils perçoivent que de par sa structure, son fonctionnement et ses ressources, la HEP-BEJUNE leur offre probablement de meilleures conditions et de meilleures chances de réussite dans ces visées que dans d'autres. Leur mémoire pourra certainement répondre à la triple exigence de clarté, de pertinence et de faisabilité attendue (Quivy et van Campenhoudt, 1995), dans le temps qui leur est imparti. Pour ce faire, ils bénéficient :

- d'un savoir déjà acquis durant leur cursus et d'un savoir-faire déjà acquis durant les stages au cours de leurs deux premières années de formation dans ces domaines;

- d'un guidage, d'une guidance ou d'un accompagnement (Bailleul, 2005) d'un directeur de mémoire souvent expert dans ces domaines de par son profil professionnel; 
- d'un accès balisé à une littérature abondante et déjà partiellement intégrée dans ces domaines dans les cours de didactique et de sciences de l'éducation;

- d'un terrain de récolte de données à portée de main (une classe de stage) qu’ils connaissent déjà et dans lequel ils peuvent anticiper les difficultés de mise en œuvre de leur méthodologie;

- de l'aide d'un formateur en établissement qui met à disposition sa classe et les épaule durant la récolte des données;

- de la connaissance des élèves qu'ils vont tester, interroger ou observer, puisque les étudiants sont aussi leur enseignant stagiaire référent lorsqu'ils mettent en œuvre leur recherche.

La motivation des étudiants à développer des connaissances et des compétences professionnelles centrées sur les disciplines scolaires, leur didactique et leurs dispositifs témoigne que ces novices ${ }^{4}$ pensent qu'elles constituent la clé de réussite de l'entrée dans la profession, conformément à la première compétence professionnelle énoncée dans le référentiel de la HEP-BEJUNE (2011) : "Concevoir et animer des situations d'enseignement et d'apprentissage en fonction des élèves et du plan d'études » (p. 9).

La seconde interprétation possible est d'ordre «identitaire ». Fabre et Lang (2000) avaient déjà relevé que, pour leur première expérience de recherche, un grand nombre d'étudiants aimeraient donner à voir « les ajustements qu'ils [seront capables de produire] entre leur vision de l'institution [scolaire] et leur avenir possible» (p. 52). Aussi, beaucoup d'étudiants de la HEP-BEJUNE affichent cette même identité professionnelle provisoirement assumée dans leur choix majoritairement disciplinaire ou portant sur des éléments de gestion des apprentissages. En effet, réagissant à des lacunes constatées dans le terrain qu'ils pensent pouvoir combler et motivés par une valorisation d'eux-mêmes en cas de succès, ils espèrent bouleverser l'ordre établi en mettant à l'épreuve de la réalité de la classe une séquence d'enseignement/apprentissage qu'ils jugent innovante dans une discipline donnée. Dans certains cas, l'expertise d'un étudiant dans une discipline spécifique lui semble suffisamment développée - parfois davantage que ses propres formateurs en établissement - pour l'autoriser à tester un moyen qu'il juge novateur. On retrouve des manifestations de cet ordre dans certaines introductions des mémoires analysés :

Étant musicienne de première formation, le sujet de la musique à l'école a forcément une place particulière pour moi [...]. Raison pour laquelle je me demande si elle ne devrait pas être plus présente dans le domaine scolaire. J'ai envie de pouvoir enseigner cette discipline pour toutes les richesses qu'elle peut apporter, sans la limiter au chant, comme le font, ainsi que j'ai pu le voir, bon nombre d'enseignants et comme m'en ont fait part plusieurs enfants autour de moi. [Fjo13]

Enfin, le score honorable (16,67 \%) de la visée Traits spécifiques des élèves (voir Figure 2) révèle que les étudiants de la HEP-BEJUNE sont très sensibles aux élèves différents, à leurs caractéristiques et à la manière de les faire progresser. Ils savent que dans une classe, il faut aussi, pour atteindre l'excellence enseignante, savoir mettre en œuvre une pédagogie différenciée au service de la progression de tous les élèves. Mais on peut aussi y voir un effet de halo, comme pour la problématique des élèves à haut potentiel ou celle de l'hyperactivité, largement débattues dans les médias ces dernières années, provoquant un regain d'intérêt au sein de la communauté enseignante. 
Cependant, au vu des résultats généraux, on pourrait se demander pourquoi, à la HEP-BEJUNE, les étudiants ne choisissent pas davantage des sujets liés à la gestion de la classe (12,5\%), une thématique phare dans les curricula des sciences de l'éducation ${ }^{5}$. Peut-être pensent-ils qu'une bonne maîtrise des contenus disciplinaires et de leur didactique sont les éléments socles de la compétence enseignante et que les problèmes de gestion de classe peuvent être évités, dès lors qu'ils font preuve de cette compétence disciplinaire et didactique? On pourrait aussi supposer que les étudiants sont moins sensibles aux problèmes de gestion de la classe, n'ayant encore jamais effectué de stages en responsabilité complète lorsqu'ils s'engagent dans leur mémoire. Peut-être encore faut-il quelques années de pratique avant de considérer que l'établissement d'une relation éducative avec l'élève est le préalable à toute progression dans les apprentissages? Nous verrons ci-après que le choix de ces visées pourrait être modulé en fonction des caractéristiques de la culture de recherche et du profil professionnel des formateurs du site dans lequel l'étudiant a été formé.

\section{Les visées de la recherche par site de production : un effet de contexte « culture de la recherche »?}

L'analyse des fréquences d'apparition des visées de la recherche dans l'échantillon (voir Figure 1) a été confrontée au regard des scores des visées de la recherche, redistribués en fonction des trois sites de production (SPO : $n=10 ; \mathrm{SBI}: n=14 ; \mathrm{SCF}: n=24$ ).

De manière générale, les fréquences d'apparition des visées de la recherche présentées sous forme de radar en fonction du site de production des mémoires (voir Figure 2) témoignent que les profils des visées de la recherche se rejoignent pour les trois sites sur les trois visées qui avaient obtenu les meilleurs scores dans les résultats généraux (voir Figure 1), à savoir :

1) la visée Disciplinaire $(\mathrm{SBI}=28,6 \% ; \mathrm{SCF}=25 \%$; $\mathrm{SPO}=15 \%$ );

2) la visée Gestion des apprentissages ( $\mathrm{SBI}=28,6 \%$; $\mathrm{SCF}=11,8 \%$; $\mathrm{SPO}=45 \%$ );

3) la visée Traits spécifiques des élèves ( $\mathrm{SBI}=7,1 \%$; $\mathrm{SCF}=22,9 \% ; \mathrm{SPO}=15 \%$ ).

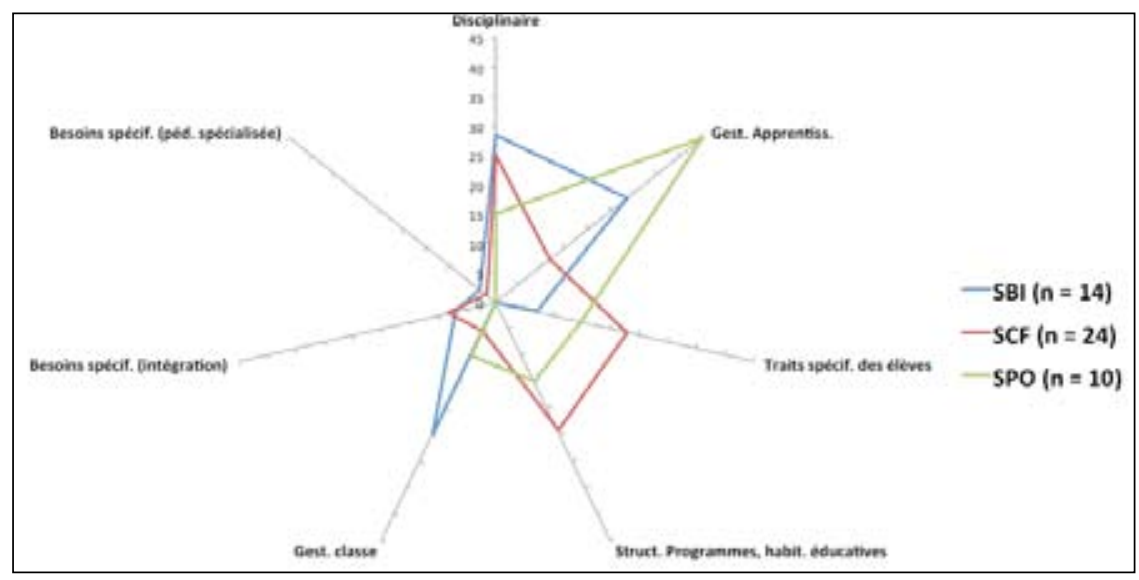

Figure 2

Radar des fréquences d'apparition des visées de la recherche en fonction du site de production des mémoires de la HEP-BEJUNE (SBI: $\mathrm{n}=14 ; S C F: \mathrm{n}=24 ; S P O: \mathrm{n}=10)$. 
Par contre, le SBI se démarque des autres sites par des visées sur la Gestion de la classe $(25 \%)$ et Disciplinaire (28,6\%). Le SCF se démarque par des visées sur Structures, programmes et habitudes éducatives et Traits spécifiques des élèves. Le SPO se démarque par des visées sur Gestion des apprentissages (45\%).

Le profil des visées du SCF est relativement comparable à celui du SPO (voir Figure 2), si nous relativisons le score SPO, Gestion des apprentissages (45\%). Un écart relativement comparable sépare leurs scores sur trois visées, Disciplinaire (10\%), Structures, programmes et habitudes éducatives (9,3\%) et Traits spécifiques (7,4\%). Le profil des visées du SPO est relativement comparable à celui du SBI, puisqu'un écart relativement comparable sépare leurs scores sur deux visées importantes, Disciplinaire $(13,6 \%)$ et Gestion de la classe (15 \%). Par contre, les profils des visées de la recherche sur le SBI et celui du SCF semblent les plus éloignés. Un écart relativement comparable sépare leurs scores pour une seule visée : Disciplinaire (3,6\%).

L'hétérogénéité des écarts à la moyenne des scores de chaque site pour les sept visées de la recherche montrent (voir Figure 3), qu'il n'y a pas de consensus quant à l'importance que chacun de ces sites semble leur attribuer.

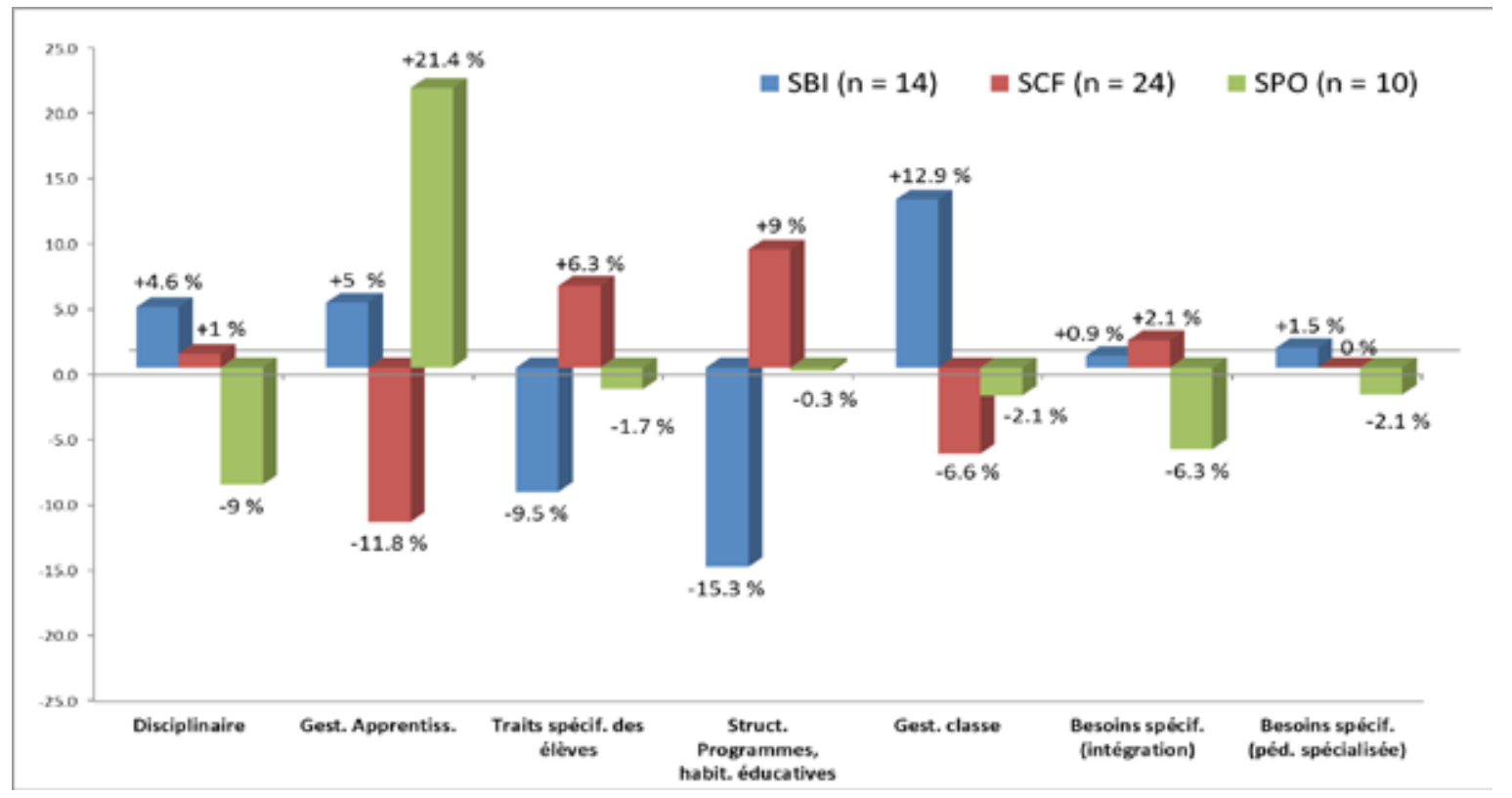

\section{Figure 3}

Écart des fréquences d'apparition des visées de la recherche par rapport à la moyenne générale en fonction du site de production des mémoires ( $S B I: \mathrm{n}=14 ; S C F: \mathrm{n}=24 ; S P O: \mathrm{n}=10)$. 
Le SBI se démarque de la moyenne des trois sites par une production plus importante de mémoires à visée Gestion de la classe (+ 12,9\%). Dans l'équilibre de ses visées prioritaires, il intègre encore la visée Gestion des apprentissages (+ $5 \%$ ) et la visée Disciplinaire (+ 4,6\%). Par contre, par rapport à la moyenne, le SBI a tendance à produire moins de mémoires à visée Traits spécifiques des élèves (- 9,5\%) et à ne pas produire de mémoires à visée Structures, programmes et habitudes éducatives (- 15,3 \%).

Le SCF se démarque de la moyenne des trois sites par une production plus importante de mémoires à visée Structures, programmes et habitudes éducatives (+ 9\%) et Traits spécifiques des élèves (+ 6,3\%). Dans l'équilibre de ses visées prioritaires, il intègre encore la visée Disciplinaire (+ $1 \%)$. Par contre, par rapport à la moyenne, il a tendance à produire nettement moins de mémoires à visée Gestion des apprentissages (- $11,8 \%)$.

Le SPO se démarque de la moyenne des trois sites par une production plus importante de mémoire à visées Gestion des apprentissages (+ 21,4\%). Dans l'équilibre de ses visées prioritaires, il intègre la visée Disciplinaire (15\%), Structures, programmes et habitudes éducatives (15\%) et Traits spécifiques des élèves (15\%) (voir Figure 2), sans pour autant qu'elles se démarquent significativement de la moyenne des autres sites (voir Figure 3). Par contre, par rapport à la moyenne, il a tendance à produire moins de mémoires à visée Disciplinaire (- 9\%) et à visée Besoins spécifiques (intégration) (- 6,3\%).

\section{Discussion}

Au vu de la faible distribution des mémoires par site de formation de notre échantillon, nous ne pouvons qu'esquisser des pistes interprétatives quant à ces disparités constatées dans la distribution des visées des recherches. La production importante de travaux relatifs à la gestion de la classe suggère que la culture de recherche du SBI serait orientée davantage vers les sciences de l'éducation. Celle du SCF davantage vers les disciplines, mais avec un accent fort sur des éléments psychologiques et structurels. Enfin le SPO, en privilégiant les visées sur la gestion des apprentissages montreraient sa propension à insister sur des éléments didactiques, du point de vue métacognitif.

Rappelons que chacun des trois sites a organisé sa formation en recherche sur le même nombre de crédits, distribués de manière identique sur les trois années de formation, avec les mêmes prescriptions sur les contenus de cours et avec un règlement commun pour la rédaction du mémoire et la procédure d'évaluation écrite et orale. Partant du principe que les étudiants ont encore pu choisir librement leur sujet, ces disparités pourraient révéler une influence plus importante que ce que l'on pourrait attendre. D'une part, elles seraient à mettre sur le compte du profil professionnel des formateurs qui ont piloté la formation générale en recherche et, d'autre part, sur le profil professionnel des directeurs de mémoires qui ont encadré le mémoire. Au vu de la difficulté d'intégrer la recherche dans une formation d'enseignant qui n'y était pas habituée, cette formation en recherche BEJUNE a été portée par des formateurs chevronnés et des directeurs de mémoire fortement imprégnés de la culture de leurs sites respectifs. Ils ont implicitement retraduit ces prescriptions en vision incarnée et parfois militante en faveur de leurs domaines de prédilection, pour mieux stabiliser ce nouveau processus de formation dans l'institution, mais aussi pour garantir une meilleure affiliation des étudiants au parcours de la recherche. 


\section{Conclusion}

L'étude des visées de la recherche contenues dans les $\mathrm{QR}$ des mémoires de bachelor produits par les étudiants de la HEP-BEJUNE a permis de faire émerger deux constats, selon le regard que nous avons porté sur l'étudiant, sur le site de production ou sur les directeurs.

Le premier constat porte sur les objectifs manifestes de recherche que les étudiants de la HEPBEJUNE entendent atteindre en rédigeant un mémoire : en choisissant de produire un mémoire à visée disciplinaire ou qui traite de la gestion des apprentissages, ils s'inscrivent prioritairement dans une logique de recherche pédagogique pragmatique, liée à des impératifs praxéologiques. Ils espèrent mettre à profit, pour eux-mêmes d'abord, la plus-value offerte par le parcours et les résultats de leur mémoire. Mais l'analyse de ces choix suggère aussi que les étudiants pensent qu'ils bénéficieront de conditions favorables en termes de ressources, de moyens et d'encadrement pour produire un mémoire qui répondra aux attentes institutionnelles. Pour certains, c'est aussi l'occasion d'afficher une identité professionnelle provisoirement assumée en essayant de démontrer qu'ils seront capables de produire une innovation dans une discipline dans laquelle ils s'estiment experts et dans laquelle ils ont l'impression que des lacunes sont à déplorer sur le terrain.

Le second constat porte sur les objectifs latents de recherche que la formation de la HEP-BEJUNE entend atteindre à travers la production de ses mémoires de bachelor. Malgré la volonté affichée d'uniformiser la structure et les contenus de formation, chaque site présente des particularités d'enseignement de la recherche, des particularités de style d'encadrement et de contenus à produire qui modulent l'importance prioritaire des visées, redistribuées en fonction des sites de production. Nous suggérons que les profils professionnels des formateurs, spécifiques pour chacun des trois sites en fonction de leur histoire respective, ont créé en effet « culture de recherche » intrasite qui a orienté les visées des recherches de manière plus importante que ce qui était attendu. Une formation en recherche et ses produits se définissent, en fin de compte, par le profil des acteurs qui la portent et le profil des acteurs qui la suivent.

Concernant les objectifs manifestes des étudiants liés à une logique de recherche pédagogique pragmatique, nous pensons qu'ils peuvent parfaitement se justifier et même être encouragés, en tant que plus-value dans une formation qui forme des enseignants novices, dont la mission première est d'enseigner, même si ces objectifs ne réalisent pas complètement la prise de distance objectivante attendue, créée par la suspension partielle de l'action; des intérêts déliés de la pratique qui pourront se manifester de manière plus aigüe dans une formation continue en recherche pour des enseignants chevronnés.

Concernant les objectifs latents de la HEP-BEJUNE d'uniformiser la formation en recherche, plutôt que de souhaiter qu'un mémoire, à ce jour réalisé dans une forme de projet conjoint ou collaboratif entre l'étudiant et son directeur, ne se sclérose dans une forme trop prescriptive, voire administrative, il est probable qu'un bon mémoire restera toujours un objet polymorphe, négocié entre les représentations et les attentes des étudiants, les attentes institutionnelles et les attentes des directeurs de mémoire, en fonction de leurs compétences spécifiques. Un équilibre instable et toujours à reconstruire, à l'instar de la recherche vivante. 


\section{Références}

Altet, M. (2000). L'analyse de pratiques : une démarche de formation professionnalisante? Recherche et formation, 35(1), 2541. http://dx.doi.org/10.3406/refor.2000.1668

Arcidiacono, F. et De Gregorio, E. (2008). Methodological thinking in psychology : Starting from mixed methods. International Journal of Multiple Research Approches, 2(1), 118-126. http://dx.doi.org/10.5172/mra.455.2.1.118

Bailleul, M. (2005). L'accompagnement du mémoire professionnel en formation d'enseignants : un « bout de chemin ». Formation et pratiques d'enseignement en questions, (2), 11-26. Repéré à http://www.revuedeshep.ch/site-fpeq-n/Site FPEQ/2 files/2005-2-Bailleul.pdf

Bailleul, M. et Bodergat, J.-Y. (2001). Diriger un mémoire professionnel : entre dispositif institutionnel et problématique relationnelle. Le cas de la formation des enseignants en France. European Journal of Teacher Education, 24(3), 263289. http://dx.doi.org/10.1080/02619760220128851

Bourdoncle, R. (1994). L'université et les professions : un itinéraire de recherche sociologique. Paris : L'Harmattan.

Clanet, J. (2010). Professionnalisation des métiers de l'enseignement : l'apport de l'observation des pratiques. Recherche en éducation, (8), 73-84. Repéré à http://www.recherches-en-education.net/IMG/pdf/REE-no8.pdf

Conférence suisse des directeurs cantonaux de l'instruction publique (CDIP). (2001). Déclaration de la CDIP relative aux perspectives professionnelles dans l'enseignement. Repéré à http://edudoc.ch/record/25527/files/20010618Leh_f.pdf

Crinon, J., Marin, B., Rayou, P. et Ricard-Fersing, E. (2005). Le mémoire professionnel : pour un accompagnement efficace. Repéré à http://ressources.creteil.iufm.fr/fileadmin/documents/siteFFO/Service/Productions/2005 Memoire pro.pdf

Cros, F. (dir.) (1998). Le mémoire professionnel en formation des enseignants : un processus de construction identitaire. Paris : L'Harmattan.

Dépelteau, F. (2000). La démarche d'une recherche en sciences humaines : De la question de départ à la communication des résultats. Bruxelles : De Boeck.

Fabre, M. et Lang, V. (2000). Le mémoire professionnel IUFM est-il professionnalisant? Recherche et formation, 35(1), 4358. http://dx.doi.org/10.3406/refor.2000.1669

Giroux, S. et Tremblay, G. (2009). Méthodologie des sciences humaines : la recherche en action (3éd.). Québec, QC : ERPI.

Haute école pédagogique BEJUNE (HEP-BEJUNE). (2006). Une institution de niveau tertiaire au service de la pédagogie : bilan 2001 à 2006 (Rapport quinquennal du rectorat). Repéré à http://edudoc.ch/record/24697/files/Rapport quinquennal V1.pdf

Haute école pédagogique BEJUNE (HEP-BEJUNE). (2010). Directives relatives au mémoire de bachelor pour les étudiantes et étudiants en formation préscolaire et primaire. Porrentruy : HEP-BEJUNE.

Haute école pédagogique BEJUNE (HEP-BEJUNE). (2011). Référentiel de compétences pour la formation initiale des enseignants du niveau primaire de la HEP-BEJUNE et son utilisation pour la conception du programme de formation (Comité programme). Porrentruy : HEP-BEJUNE.

Heer, S. et Akkari, A.-J. (2005). L'arroseur arrosé : les bénéfices insoupçonnés de la direction des mémoires professionnels. Formation et pratiques d'enseignement en questions, (2), 109-123. Repéré à http://www.revuedeshep.ch/site-fpeq-n/Site FPEQ/2 files/2005-2-Heer.pdf

Hofstetter, R. (2005). La «professionnalisation» des enseignants à travers une initiation à la recherche : le mémoire comme formation à et par la recherche de la Section des sciences de l'éducation de l'Université de Genève. Formation et pratiques d'enseignement en questions, (2), 71-89.

Hofstetter, R. et Schneuwly, B. (dir.) (2009). Savoirs en (trans)formation : au cour des professions de l'enseignement et de la formation. Bruxelles : De Boeck.

Joliat, F. (2011). Un mémoire de recherche collaborative basé sur l'observation systématique des comportements musicaux. Dans F. Joliat (dir.), La formation des enseignants en musique : état de la recherche et vision des formateurs (p. 57-75). Paris : L'Harmattan. 
Lamoureux, A. (2006). Recherche et méthodologie en sciences humaines (2 éd.). Montréal, QC : Beauchemin.

Lang, V. (2004). La profession enseignante en France : permanence et éclatement. Dans M. Tardif et C. Lessard (dir.), La profession d'enseignant aujourd'hui : évolutions, perspectives et enjeux internationaux (p. 157-171). Bruxelles : De Boeck.

Paillé, P. et Mucchielli, A. (2008). L'analyse à l'aide des catégories conceptualisantes. Dans P. Paillé et A. Mucchielli (dir.), L'analyse qualitative en sciences humaines et sociales (2 éd., p. 233-272). Paris : Colin.

Perrin, D., Bailleul, M. et Bodergat, J.-Y. (2009). Le mémoire professionnel pour les étudiants de la Haute Ecole Pédagogique de Berne, Jura et Neuchâtel : entre constitution d'outils et démarche compréhensive. Dans M. Mellouki et A. Akkari (dir.), La recherche au service de la formation des enseignants (p. 13-41). Repéré à http://doc.rero.ch/record/234548

Perrin, N., Bétrix Köhler, D., Martin, D. et Baumberger, B. (2008). Le mémoire professionnel : un moyen pour évaluer les compétences des étudiants à l'enseignement. Dans L. Lafortune, S. Ouellet, C. Lebel et D. Martin (dir.), Réféchir pour évaluer des compétences professionnelles à l'enseignement : deux regards, l'un québécois et l'autre suisse (p. 157-178). Québec, QC : Presses de l'Université du Québec.

Quivy, R. et van Campenhoudt, L. (1995). Manuel de recherche en sciences sociales (2 ${ }^{\mathrm{e}}$ éd.). Paris : Dunod.

Tardif, M. et Borges, C. (2009). L'internationalisation de la professionnalisation de la formation à l'enseignement secondaire et ses retraductions dans des formes sociales nationales. Dans R. Hofstetter et B. Schneuwly (dir.), Savoirs en (trans)formation: au cceur des professions de l'enseignement et de la formation (p. 109-136). Bruxelles : De Boeck. http://dx.doi.org/10.3917/dbu.hofst.2009.01.0109

Tardif, M. et Lessard, C. (dir.) (2004). La profession d'enseignant aujourd'bui : évolutions, perspectives et enjeux internationaux. Bruxelles : De Boeck.

Vanhulle, S. (2005). Écriture réflexive et subjectivation de savoirs chez les futurs enseignants. Nouveaux cabiers de la recherche en éducation, 8(1), 41-63. http://dx.doi.org/10.7202/1018157ar

Vanhulle, S. (2009a). Évaluer la professionnalité émergente des futurs enseignants : un pari entre cadres contraignants et tensions formatives. Dans L. Mottier Lopez et M. Crahay (dir.), Évaluations en tension (p. 165-180). Bruxelles : De Boeck. http://dx.doi.org/10.3917/dbu.motti.2009.01.0165

Vanhulle, S. (2009b). Dire les savoirs professionnels : savoirs de référence et logiques d'action. Dans R. Hofstetter et B. Schneuwly (dir.), Savoirs en (trans)formation: au cour des professions de l'enseignement et de la formation (p. 245-263). Bruxelles : De Boeck. http://dx.doi.org/10.3917/dbu.hofst.2009.01.0245

Wentzel, B. (2010). Le praticien réflexif : entre recherche, formation et compétences professionnelles. Dans B. Wentzel et M. Mellouki (dir.), Recherche et formation à l'enseignement : spécificités et interdépendance (p. 15-35). Repéré à http:// doc.rero.ch/record/234561/files/DC ActesRecherche 8 Recherche et formation a 1 enseignement 16 36.pdf

Wentzel, B. (2012). Réflexivité et formation professionnelle des enseignants : actualités d'un paradigme en construction. Dans M. Tardif, C. Borges et A. Malo (dir.), Le virage réflexif en éducation. Où sommes-nous 30 ans après Schön? (p. 143-160). Bruxelles : De Boeck. http://dx.doi.org/10.3917/dbu.tardi.2012.01.0143

Wittorski, R. (2008). Professionnalisation et offres de formation dans l'enseignement supérieur. Dans C. Solar et P. Hébrard (dir.), Professionnalisation et formation des adultes : une perspective universitaire France-Québec (p. 59-87). Paris : L'Harmattan.

\section{Notes de bas de page}

1 Dans le présent document, les termes employés pour désigner des personnes sont pris au sens générique; ils ont à la fois valeur d'un féminin et d'un masculin.

2 Ces profils correspondent bien aux formateurs concernés par notre étude. Mais à l'heure actuelle, le profil universitaire des formateurs de la HEP-BEJUNE est certainement plus important.

3 Seule, la proportionnalité entre le nombre de mémoires fournis par année et par site n’a pas été strictement respectée. Nous observons, par exemple, que pour l'année 2008, le total des mémoires analysés est de 10, alors que la production totale s'élevait à 66. Pour l'année 2008, elle est de 4 seulement, bien que la production totale se soit élevée à 77. 
$4 \quad$ Il serait intéressant de mener une étude sur les visées de la recherche en comparant nos résultats avec des mémoires produits par des enseignants chevronnés ayant repris une formation certifiante après plusieurs années passées sur le terrain. Nous pourrions peut-être observer dans quelle mesure leurs intérêts se sont déplacés vers des éléments davantage liés à la relation éducative, au contexte, à l'organisation et aux structures scolaires.

5 Nous verrons plus loin que cette remarque ne concerne en réalité que les mémoires produits sur le SPO et le SCF.

\section{Pour citer cet article}

Joliat, F. et Arcidiacono, F. (2016). L'analyse des visées de la recherche dans la production des mémoires de bachelor de la HEP-BEJUNE : une question d'intérêt professionnel et de culture(s) de recherche(s)? Formation et profession, 24(2), 17-32. http://dx.doi.org/10.18162/fp.2016.344 